\title{
The Symmpact: A Direct-Impact Hopkinson Bar Setup Suitable for Investigating Dynamic Equilibrium in Low-Impedance Materials
}

\author{
P. Jakkula ${ }^{1} \cdot$ G. C. Ganzenmüller ${ }^{1,2}\left(\mathbb{D} \cdot\right.$ S. Beisel ${ }^{1} \cdot$ P. Rüthnick ${ }^{2} \cdot$ S. Hiermaier ${ }^{1,2}$
}

Received: 24 March 2021 / Accepted: 25 September 2021 / Published online: 7 October 2021

(c) The Author(s) 2021

\begin{abstract}
Background Measuring the dynamic behavior of low-impedance materials such as foams is challenging. Their low acoustic impedance means that sensitive force measurement is required. The porous structure of foams also gives rise to dynamic compaction waves, which can result in unusual behavior, in particular if the foam material is so thick, that dynamic force equilibrium is not reached.

Objective This work investigates comparatively large polyurethane foam specimens with densities in the range of $80-240$ $\mathrm{kg} / \mathrm{m}^{3}$ to deliberately achieve a state away from force equilibrium during high-rate compaction. The aim is to understand how an increase in strain rate leads to a reduction in strength for such materials.

Methods A specialized direct-impact Hopkinson bar is employed. It uses polycarbonate bars to achieve the required long pulse duration of $2.6 \mathrm{~ms}$ to compress the large specimens into the densification regime. In contrast to existing setups, both striker and output bar are instrumented with strain gauges to monitor force equilibrium. The absence of an input bar allows monitoring force equilibrium more accurately. Special attention is paid to the calibration of strain gauges, taking non-linear effects, wave dispersion and attenuation into account. Digital Image Correlation is employed to analyze elastic and plastic compaction waves by means of Lagrange diagrams.

Results Depending on density, the specimens show saturation of dynamic strength increase at high rates of strain $\approx 500 / \mathrm{s}$, or even negative strain rate sensitivity in case of the lowest density. The occurrence of apparent negative strain rate sensitivity is accompanied by a localized structural collapse front, moving at a low velocity of $\approx 10 \mathrm{~m} / \mathrm{s}$ through the material. This apparent strain rate sensitivity is a structural effect which is related to the thickness of the specimen.

Conclusions The primary aim of material characterization using Hopkinson bars is to achieve a state of force equilibrium. For this reason, very thin specimens are usually employed. However, data gathered in this way is not representative for thick foam layers. Here, an increase of strain rate can lead to a decrease of strength if homogeneous deformation is replaced by a dynamic compaction wave. This behavior can occur at strain rates encountered under conditions such as automotive crash.
\end{abstract}

Keywords Polyurethane foam $\cdot$ Dynamic testing $\cdot$ Split hopkinson methods

\section{Introduction}

Dynamic testing of soft materials with low-density and little stiffness has traditionally been challenging [1]. The root of the problem causing the difficulty is that dynamic

G. C. Ganzenmüller

georg.ganzenmueller@inatech.uni-freiburg.de

1 Albert-Ludwigs-Universität Freiburg, Sustainable Systems Engineering, INATECH, Emmy-Noether Str. 2, Freiburg 79110, Germany

2 Fraunhofer Institute for High-Speed Dynamics, Ernst-Mach-Institut, EMI, Ernst-Zermelo Str. 4, Freiburg 79104, Germany equilibrium of force is required for accurate force measurements during the experiment. This means that the stress state measured on either side of the specimen during uniaxial loading must be equal if the measurement is going to be representative of the bulk material behavior. Naturally, during impact loading of a specimen emanating from one side only, the stress state requires a certain time to propagate through the specimen and establish dynamic equilibrium. This time depends on the specimen's dimensions, and its elastic wave speed, $c_{0}$. Additionally, the ratio of acoustic impedances, $z=\rho c_{0}$, where $\rho$ is the mass density, between the specimen and its mechanical boundaries plays a role, as elastic wave transmission and reflection effects depend on this ratio [2]. 
Here, we are concerned with a modification of the split Hopkinson pressure bar (SHPB) [3], where the specimen is sandwiched between two slender bars. In the predominately used configuration of this setup due to Kolsky [4], a third bar, the so-called striker, impacts the first bar, which is termed the input bar. An elastic wave is created due to the impact and propagates via the input bar through the specimen, and into the output bar. Impedance differences between bar material, typically a metal, and the specimen, cause wave amplitude changes and effect compression of the specimen. The stress state in the input and output bars are inferred from strain gauge measurements, assuming a constant Young's modulus. From the stress in the bars, the forces acting on the specimen are calculated.

This approach has proven extremely successful for the dynamic characterization for metallic materials. However, it is limited in applicability to low-impedance materials for two reasons: the small force amplitude due to low specimen strength is challenging to resolve accurately on the comparatively stiff metallic bars, and the large impedance mismatch between specimen and bar material necessitates long equilibration times before accurate force readings can be obtained.

These problems have been addressed by switching from metallic bars to polymer bars. The smaller stiffness of polymers compared to metals reduces both the impedance mismatch and increases the force measuring sensitivity. However, the pronounced viscoelastic nature of most polymers means that data analysis becomes more involved as wave dispersion and attenuation need to be accounted for [5]. A review summarizing the use of polymeric bars for split Hopkinson application can be found in [6], and a general review of high rate testing in [7]. While metal bars, in particular hollow bars with semiconductor strain gauges, can also offer high force sensitivity, the low speed of sound in polymer bars yields the advantage of longer pulse duration such that larger specimens can be studied. Another approach to increase force measuring sensitivity is to use piezoelectric force transducers in between the specimen and the bars [8]. This method is in principle superior to using strain gauges on polymer bars, as the viscoelastic nature of the polymer bars does not need to be addressed. However, calibration of the piezoelectric transducers is also not trivial.

The approach presented here is based on a direct-impact Hopkinson bar. Here, no separate striker is required as the input bar itself is accelerated towards the specimen and serves as the striker. We also employ polymer bars but show that the choice of polycarbonate, together with short distances between strain gauges and specimen/bar interface, leads to negligible errors in the force measurement due to wave attenuation and dispersion. Additionally, the absence of a dedicated striker means that two nearly identical waves travel into the input and output bars. Thus, measurement of the evolution of dynamic force equilibrium becomes more accurate because wave superposition between the incident and reflected wave, as present in the classic SHPB, does not pose a problem. We note that we are not the first to pursue the general idea of a direct-impact Hopkinson bar using a striker that is instrumented with a strain gauge: Govender and Curry [9] presented this layout before with polymer bars. Hiermaier and Meenken [10] employed a metal input bar but introduced the idea of using piezoelectric foil gauges on both sides of the specimen to enhance force resolution in a direct impact configuration. Lea and Jardine measured the stresses in the input and output bar using photon Doppler velocimetry and concluded that direct-impact Hopkinson pressure bar systems offer many potential advantages over split Hopkinson pressure bars, including access to higher strain rates, higher strains for equivalent striker velocity and system length, lower dispersion, and faster achievement of force equilibrium [11].

This work builds on the ideas of these authors and gives detailed construction advice to handle the problem of guiding the input bar uni-axially while providing access to the electrical strain gauge connection.

For demonstration purposes, we investigate polyurethane (PUR) foams with different densities in the range of $80-240$ $\mathrm{kg} / \mathrm{m}^{3}$. We intentionally consider specimens with a length of $20 \mathrm{~mm}$, which is far too long by SHPB standards, as this length implies that significant time is required to reach equilibrium. Therefore, we do not study a pure material behavior, but a structural effect. For foams, it can be argued that these are structured materials for any size of specimen. Therefore, measured mechanical properties will depend on the size of the specimen and hence cannot straightforwardly be extrapolated to full-scale objects. Our specimens exhibit the peculiar behavior of saturation of dynamic strength increase at high rates of strain $\geq 500 / \mathrm{s}$, or even negative strain rate sensitivity in case of the lowest density. The direct-impact Hopkinson bar presented here is well suited to investigate this phenomenon as it features a long pulse duration. Additionally, the high force sensitivity allows the development of dynamic force equilibrium to be accurately studied, or even whether it is achieved. We link the observed features in the force signals to an analysis of strain rate distribution over the specimen. This approach indicates that the apparent negative strain rate sensitivity is accompanied by a structural collapse front.

The literature provides ample examples of foam studies, where interesting behavior for foams, which can be considered a structured material, is observed: Koumlis and Lamberson [12] study a PUR foam with a density of 57 $\mathrm{kg} / \mathrm{m}^{3}$ using both polycarbonate and aluminum bars in a modified Hopkinson bar setup. Thin specimens of length $3 \mathrm{~mm}$ are considered, with the intention of observing a volume representative of the bulk material behavior. A logarithmic increasing strain rate dependency of the yield 
strength is observed. Due to the small length, equilibrium is achieved, and no inhomogeneous collapse front is observed. The authors mention that heterogeneous behavior and the effects of a relaxation of stress equilibrium are worthwhile to investigate.

Barnes et al. [13] investigated compaction of open-cell aluminum foams up $160 \mathrm{~m} / \mathrm{s}$. For velocities lower than 40 $\mathrm{m} / \mathrm{s}$, specimen response was similar to the quasi-static case. For higher velocities, the plateau stress increased proportional to the square of the impact velocity. A critical impact velocity was identified, at which the compaction behavior changes from a homogeneous nature to the propagation of a shock-like collapse front.

Ravindran et al. [14] investigated a closed cell polymer foam with density of $\approx 150 \mathrm{~kg} / \mathrm{m}^{3}$. Similar to the work of Barnes et al., digital image correlation was used to identify the speed of an elastic precursor and a much slower shocklike compaction front. The work presented here shows similarities to these studies, in particular in terms of optical data analysis. However, we focus on lower velocities and link the occurrence of non-homogeneous compaction to the lack of force equilibrium within the specimen. In contrast to the gasgun direct-impact configuration of the afore cited studies, our setup allows measurement of the force on the impact side of the specimen. Further relevant studies include [15-17].

The remainder of this work is organized as follows. In Materials and Methods we first describe the PUR foam specimens and the conventional testing machine used to obtain their quasi-static and low-strain rate properties. Then, the mechanical components of the Symmpact are described, along with details of the electronic data acquisition system. Dynamic Data Analysis shows the post-processing of Symmpact data, including the effects of viscoelasticity in the bars. Results then proceeds to the analysis of strain-rate sensitivity for the PUR foams and investigates in detail their dynamic compaction behavior. Finally, the findings are summarized and discussed with respect to existing works.

\section{Materials and Methods}

\section{Specimens}

Polyurethane foams (Sikablock M80, M150, M330, Sika Germany) were obtained in thick plates of dimensions $150 \times 120 \times 50 \mathrm{~mm}^{3}$. These foams are typically used for building prototype models and molding forms due to their excellent machining properties. The foams are made by mixing isocyanates with polyols and small amounts of water, resulting in a cross-linking reaction and the production of $\mathrm{CO}_{2}$ gas, creating bubbles. The foams here are rigid and composed of a mixture of both closed-cell and open-cell structures. Their values for density $\rho$, Young's modulus $E$, crush strength $\sigma$, and speed of sound $c_{0}=\sqrt{E / \rho}$ according
Table 1 Physical and quasi-static mechanical properties of the foams investigated

\begin{tabular}{lllll}
\hline foam & $\rho\left[\mathrm{g} / \mathrm{cm}^{3}\right]$ & $E[\mathrm{MPa}]$ & $\sigma[\mathrm{MPa}]$ & $c_{0}[\mathrm{~m} / \mathrm{s}]$ \\
\hline M80 & 0.08 & 24 & 0.8 & 547 \\
M150 & 0.15 & 65 & 2.2 & 658 \\
M330 & 0.24 & 150 & 4.0 & 790 \\
\hline
\end{tabular}

to the manufacturer are summarized in Table 1. Testing specimens were obtained as right cylinders with diameter $30 \mathrm{~mm}$ and height $20 \mathrm{~mm}$ from foam plates by CNC milling. Scanning electron microscope images are shown in Fig. 1, showing a transition from predominantly open cell to closed cell structures as density increases.

\section{Quasi-Static and Low Strain Rate Testing}

Compression tests at $\dot{\varepsilon}=0.0017$ /s and $\dot{\varepsilon}=0.5$ /s were performed using a universal testing machine (Zwick-Roell Z100) equipped with a $100 \mathrm{kN}$ load cell of accuracy class 1 for forces $\geq 200 \mathrm{~N}$. Specimens were placed on rigid steel compression plates. The nominal strain was calculated from the displacement of the compression plates, measured using Digital Image Correlation (DIC, GOM Correlate, Germany) by tracking optical markers. The accuracy of the nominal strain measurement is better than $0.1 \%$.

\section{The Symmpact Testing Device}

The Symmpact testing device implements a direct-impact Hopkinson bar with an instrumented striker, which also serves as an input bar. The design challenge is to provide a pneumatic acceleration barrel that restricts the input bar's motion to one direction only and provides space for strain gauges and their wiring. We have solved this by utilizing a hollow aluminum extrusion profile of length $2800 \mathrm{~mm}$ with an internal diameter $\varnothing=40 \mathrm{~mm}$. The input bar of length $2000 \mathrm{~mm}$ is placed into this profile, and pressurized air is used to accelerate the input bar towards the specimen placed on the output bar. Figure 2 provides a sketch indicating the setup and the dimensions. Two slots are cut into the profile to create the space needed for the strain gauges, as detailed in Fig. 3. Concentric alignment of the

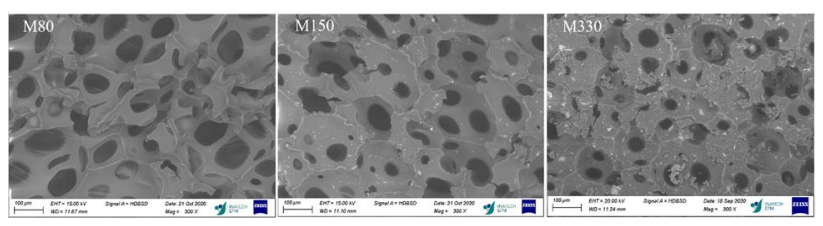

Fig. 1 Scanning electron images of the freshly milled surfaces of M80, M150 and M330 foam 


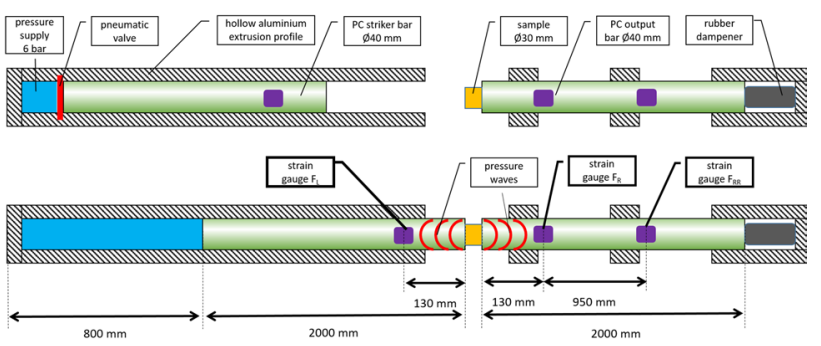

Fig. 2 Sketch of the direct-impact Hopkinson bar used in this work

two profiles attained by constraining their relative lateral and angular placement during assembly with a precision-ground straight steel shaft $(\varnothing 40 \mathrm{~mm}, \mathrm{~h} 6$ tolerance, $0.1 \mathrm{~mm}$ straightness $/ 1000 \mathrm{~mm}$ ) of $3 \mathrm{~m}$ length, protruding $1.5 \mathrm{~m}$ in each profile.

The extrusion profile is an off-the-shelf component (ITEM, Germany) with a tolerance of the internal bore of $+0.1 /-0 \mathrm{~mm}$. Both input and output bars are made from $\varnothing 45 \mathrm{~mm}$ polycarbonate (PC) stock and turned on a lathe to precisely fit the bore with little friction. To guide the output bar, short cutoffs from the same aluminum profile are used. The acceleration distance is $800 \mathrm{~mm}$, and we require an overpressure of 3 bar to achieve an input bar velocity of $12 \mathrm{~m} / \mathrm{s}$. This setup provides a usable test duration of $2.6 \mathrm{~ms}$ due to the large length of the bars and the low speed of sound in PC, such that comparatively large specimens can be tested.

\section{Dynamic Data Acquisition}

\section{Force Measurement}

Forces associated with the elastic waves in the PC bars are calculated from strain gauge measurements. At each of the three gauge stations depicted in Fig. 2, two strain gauges are placed diametrically opposite on the bar to

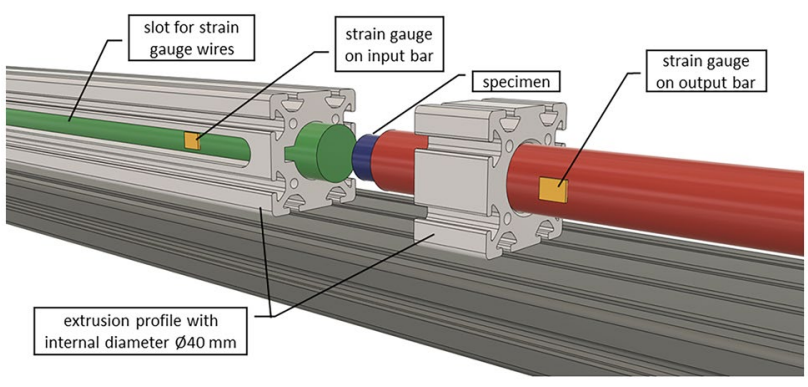

Fig. 3 Detail of strain gauge access. The hollow extrusion profile guiding the input bar features slots that provide access for the strain gauge wires (not shown here) compensate for bending; see Table 6, circuit Nr. 5 in [18]. The Wheatstone bridge signal is amplified by a factor of 100 using an amplifier with $1.5 \mathrm{MHz}$ bandwidth (Elsys SGA-2, Switzerland) and digitally stored using a transient recorder with 14-bit resolution (Elsys TPCE, Switzerland). To convert the raw voltages into forces, we first perform a quasi-static calibration of the entire Symmpact setup: the input bar is fixed on the left side, a known calibrated force sensor with $20 \mathrm{kN}$ capacity (KMZ-16z, ME Messsysteme, Germany) is placed between input and output bar, and the output bar is compressed against the input bar with a screw mechanism. A similar calibration procedure was presented for the SHPB in [19]. This procedure results in a calibration curve of force vs. voltage for each strain gauge station individually. The calibration curve is typically a perfectly linear function for metal bars, implying a constant Young's modulus. However, in the current case with PC bars, a quadratic relation is observed. This is in agreement with measurements of the nonlinear elastic region of PC, see e.g., [20]. We account for the nonlinearity by using a quadratic function that maps Wheatstone voltage readings $U$ to forces $F$, i.e., $F=a U+b U^{2}$. Here, $a$ and $b$ are calibration factors found by fitting this polynomial to the collected calibration data.

\section{High-Speed Imaging}

Video imaging of the foam specimens under dynamic compression is realized with a high-speed camera (Photron SA-Z 2100K), operating at $360 \mathrm{kHz}$ and a resolution of 256 $\times 96 \mathrm{px}$. The camera field of view (FOV) is $22.9 \times 8.5 \mathrm{~mm}^{2}$. High intensity LED lighting $(100 \mathrm{~W})$ in combination with a $100 \mathrm{~mm}$ macro lens was employed. A speckle pattern with an average speckle size of approximately 5 pixels diameter, was applied using an airbrush with a coarse nozzle. The video data is post-processed with DIC software (GOM Correlate, Germany). A facet size of 15 pixels and a step size of 12 pixels was used. The actual field-of-view used for the DIC computation of the strain field is $17 \times 5 \mathrm{~mm}^{2}$, which is smaller than the visible specimen area because the DIC algorithm employed only provides results within an interior window of the FOV, the size of which depends on the chosen facet size.

To accurately monitor the displacement of the specimen/ bar interfaces, an additional line-scan camera is used (JAI SW-4000M-PMCL-F). The line scan camera is advantageous compared to the area camera for this purpose, as it has a larger resolution, $4096 \mathrm{px}$ at $200 \mathrm{kHz}$. The achieved nominal strain measurement accuracy is better than $0.1 \%$ and directly comparable to the strain measurement performed with the universal testing machine. 


\section{Dynamic Data Analysis}

A typical experiment with the Symmpact testing device produces three force transients in the bar: the force to the left, $F_{L}(t)$, and to the right of the specimen, $F_{R}(t)$, and a secondary force measurement further downstream on the right of the specimen, $F_{R R}(t)$. The accuracy of these force measurements in dynamic loading situations, relative to static loading, is affected by three dominant effects:

- strain-rate dependent stiffness of the polymer bar

- dispersion, i.e., frequency-dependent wave propagation velocity

- attenuation, i.e., frequency-dependent dampening of stress amplitude

These effects are mutually related in the theory of nonlinear viscoelasticity [21]. We begin this section by first investigating the apparent strain-rate dependent stiffness of the bar material in the time domain. Then, the frequencydomain method of Bacon [22] is used to quantify dispersion and attenuation.

\section{Strain-Rate Dependent Stiffness}

We compare the force transients $F_{R}(t)$ and $F_{R R}(t)$, calibrated in static loading, with a known accurate force measurement in dynamic impact experiments. To this end, we utilize the same load cell as for the quasi-static calibration detail in Force Measurement section, which has a dynamic bandwidth of $60 \mathrm{kHz}$. This is sufficient, as is known that the frequency spectrum in SHPB experiments on polymeric bars only extends up to typically $20 \mathrm{kHz}$ [23]. For the setup used here, we observe that $99.99 \%$ of the power spectral density is contained in the frequency range $0-16 \mathrm{kHz}$, see Fig. 4(A).

During the impact experiment, the average of the force plateaus are compared, as shown by Fig. 4(B). For impact velocities between $2 \mathrm{~m} / \mathrm{s}$ and $12 \mathrm{~m} / \mathrm{s}$, we find a maximum deviation between the dynamic load cell and the quasi-statically calibrated strain gauge signals $F_{L}$ and $F_{R}$ on the PC bars of less than $1 \%$, whereas the signal $F_{R R}$ is attenuated by $\approx 3 \%$. As shown in Fig. 2, strain gauges $F_{R}$ and $F_{R R}$ are placed $130 \mathrm{~mm}$ and $950 \mathrm{~mm}$, respectively, upstream of the impact interface. Thus, we have an effective attenuation of $\bar{\alpha} \approx 3 \% / 950 \mathrm{~mm}=$ $3.2 \times 10^{-3 \%} / \mathrm{mm}$.

These findings suggest that quasi-static, non-linear calibration of PC bars is sufficient to obtain results accurate to $1 \%$ also for the dynamic regime, without considering viscoelastic effects, but only for strain gauges placed close to the specimen/bar interface.
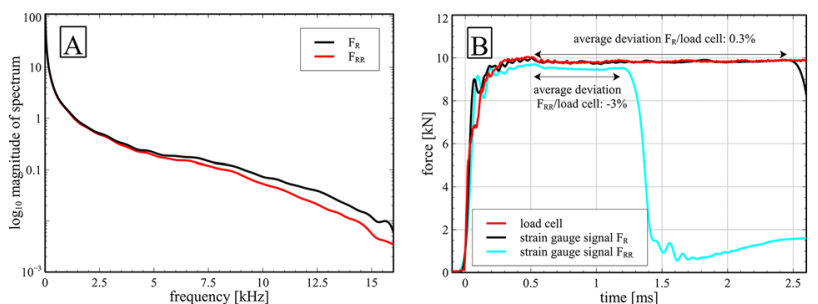

Fig. 4 (A) Magnitude of frequency spectra for $F_{R}$ and $F_{R R}$ at an impact velocity of $9 \mathrm{~m} / \mathrm{s}$ without specimen. The chosen plot cut-off of $16 \mathrm{kHz}$ encompasses $99.99 \%$ of the power spectral density (B) Validation of quasi-static strain gauge calibration for dynamic force measurement. The force obtained from the strain gauge $F_{R}$, c.f. Fig. 2 is compared against the calibrated dynamic load cell average for an impact velocity of $9 \mathrm{~m} / \mathrm{s}$. The arrows denote the period over which the average is computed

\section{Viscoelastic Wave Propagation}

Propagation of elastic waves in polymer bars is affected by both dispersion and attenuation, as the rheological properties in polymers depend on frequency. Thus, the distance at which the strain gauge is placed from the bar end interfacing with the specimen matters. The larger the distance, the more the wave is dispersed and attenuated. We remove these unwanted effects by employing the approach developed by Bacon [22], which we briefly outline below. Two wave transients, recorded at different locations in one bar, are needed. In our case, these are $F_{R}(t)$, measured at the beginning of the output bar, and $F_{R R}(t)$, measured further downstream at a distance $\Delta x$, c.f. Fig. 2 . The data used throughout this section stems from a single experiment on foam with $\rho=150 \mathrm{~kg} / \mathrm{m}^{3}$, impacted at a velocity of $10 \mathrm{~m} / \mathrm{s}$.

According to Bacon, $F_{R R}(t)$ is related to $F_{R}(t)$ by

$\hat{F}_{R R}(\omega)=\hat{F}_{R}(\omega) \exp [\gamma(\omega) \Delta x]$

$\hat{F}_{R R}(\omega)$ and $\hat{F}_{R}(\omega)$ denote the Fourier transforms of the transients $F_{R R}(t)$ and $F_{R}(t)$, and are functions of frequency $\omega$.

$\gamma(\omega)=\alpha(\omega)+i \frac{\omega}{c(\omega)}$

Equation (2) accounts for both attenuation through $\alpha(\omega)$ and dispersion through the wave speed $c(\omega)$, which both dependent on frequency. With $\hat{F}_{R R}(\omega)$ and $\hat{F}_{R}(\omega)$ known from experiment, equation (1) may be manipulated to yield:

$\alpha(\omega)=\ln \frac{\left|\hat{F}_{R}(\omega)\right|}{\left|\hat{F}_{R R}(\omega)\right|} \times \frac{1}{\Delta x}$ 
$c(\omega)=\frac{\omega \Delta x}{\theta\left[\hat{F}_{R}(\omega)\right]-\theta\left[\hat{F}_{R R}(\omega)\right]}$

Here, $\theta$ refers to the unwrapped angle of the complex-valued function $F_{R}$ or $F_{R R}$, respectively.

In practice, the computation the Fourier transforms involves some potential pitfalls that need to be avoided. It is desirable to utilize existing Fast-Fourier-Transform (FFT) routines. To ensure that FFTs of the transients $F_{R}(t)$ and $F_{R R}(t)$ are sampled at the same frequencies, and do not suffer from artifacts due to the inherent periodicity assumption of the FFT, suitable windows of these transients need to be selected. These windows need to have the same duration and number of sampling points in the time domain. We achieve this by multiplying the transients with a window function:

$W(t)=\left\{\begin{array}{l}0, \text { for } t \leq t_{0}-1.2 \mathrm{~ms} \\ 1, \text { for } t_{0}<t<t_{1} \\ 0, \text { for } t \geq t_{0}+1.2 \mathrm{~ms}\end{array}\right.$

$t_{0}$ is the time when the signal $F(t)$ begins to rise. Only 1.2 $\mathrm{ms}$ before and $1.2 \mathrm{~ms}$ after this moment are kept for the following analysis. The value of $1.2 \mathrm{~ms}$ results from the fact that the reflected wave from the right free end of the bar becomes visible in $F_{R R}(t)$ thereafter. It is only within the selected window that both $F_{R}(t)$ and $F_{R R}(t)$ show the same signal, apart from the effects of attenuation and dispersion. To illustrate this, Fig. 5(A) shows the full record of an experiment, as well as the windowed signals. The frequencydependent attenuation coefficient $\alpha(\omega)$ and the speed of sound $c(\omega)$ are found by means of equations (3) and (4) and are shown in Fig. 5(B). The speed of sound near zero frequency is $1428 \mathrm{~m} / \mathrm{s}$, which corresponds well with the frequency-independent slender bar estimate using literature values for $\mathrm{PC}, c_{0}=\sqrt{E / \rho}=\sqrt{2.4 \mathrm{GPa} / 1200 \mathrm{~kg} \mathrm{~m}^{-3}}$ $=1414 \mathrm{~m} / \mathrm{s}$. Overall, $c(\omega)$ is nearly constant for the frequency range considered here, while $\alpha(\omega)$ shows small values around $0.03 / \mathrm{m}$ near zero frequency, but becomes larger for higher frequencies. The value $\alpha(0)=0.03 / \mathrm{m}$ agrees well with the attenuation of $3 \%$ observed in Strain-Rate Dependent Stiffness section for $F_{R R}$ located at a distance of 1080 $\mathrm{mm}$ inside the bar.

Figure 5(C) shows the effect of shifting both windowed $F_{R}(t)$ and $F_{R R}(t)$, spaced apart $950 \mathrm{~mm}$, to the same location: the initial center of the specimen. The good agreement between the shifted curves shows that the simple propagation model defined by equation (1) is sufficient to account for geometric dispersion and viscoelastic effects in PC, at least for the strain rates and force amplitudes considered here. However, in our opinion, this analysis shows foremost that correcting for dispersion and viscoelastic effects is not necessary in practice for our setup. We have two strain gauges
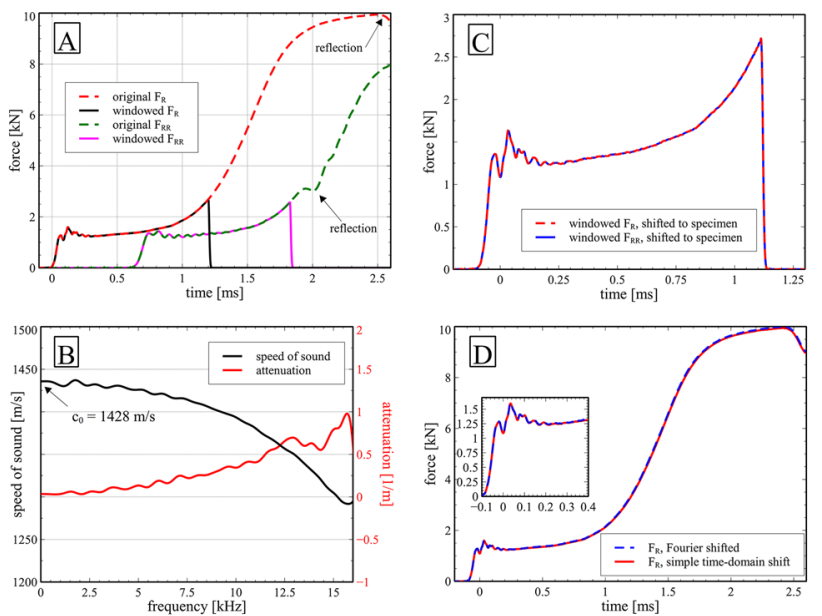

Fig. 5 (A) dashed lines are force transients measured on the output bar at two different locations. Solid lines show the windowed signals which serve as input to the Fourier-domain viscoelastic analysis. (B) frequency-dependent wave speed $c(\omega)$ and attenuation coefficient $\alpha(\omega)$ resulting from Bacon's [22] Fourier analysis. (C) windowed signals of the output bar, shifted in frequency space using Bacon's method to the center of the specimen. (D) comparison of Bacon's Fourier-space shifting method and simple time-domain shifting which considers neither wave dispersion nor attenuation

only $130 \mathrm{~mm}$ away from the specimen/bar-interfaces. For this distance, the amount of attenuation and dispersion is insignificant if an error of $1 \%$ in the force measurement is acceptable.

Figure 5(D) compares Bacon's method of shifting using equation (1) of $F_{l}(t) 130 \mathrm{~mm}$ to the left with just a simple time-domain shift using $\Delta t=130 \mathrm{~mm} / c_{0}=0.091 \mathrm{~ms}$, i.e., assuming no attenuation and no dispersion. Both signals agree to within $1 \%$ at any point the curve. For the remainder of this work, we will, therefore, only use simple time-domain shifting.

\section{Dynamic Equilibrium and Force Averaging}

The significant advantage of the Symmpact setup over conventional SHPBs lies in the fact that nearly identical stress waves propagate into both the input bar and the output bar. The only difference between these signals is that the wave in the input bar is time-shifted relative to the wave in the output bar, as the stress wave takes some time to propagate in the specimen itself. In contrast, for the SHPB, the stress wave in the input bar near the specimen is a superposition of incident wave and a reflected wave. These waves are similar in magnitude but have opposite signs and a certain noise spectrum. The superimposed signal is small due to the opposite signs, but with a larger noise spectrum, as noise is additive. The Symmpact does not suffer from this problem, as no wave superposition is present. 


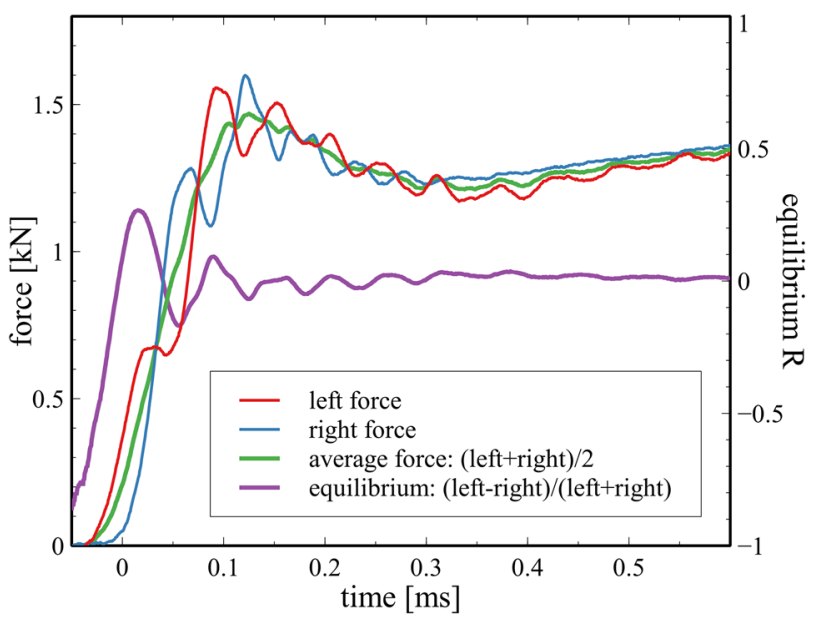

Fig. 6 Individual force signals on input and output bar and their average as well as the ratio of forces indicating force equilibrium

Figure 6 shows the evolution of force signals for an M150 foam specimen during the initial $0.6 \mathrm{~ms}$. Individual oscillations due to wave reverberation inside the specimen are very well resolved. The reverberation period matches well with the estimate, $\tau=2 L_{0} / c_{0}=0.06 \mathrm{~ms}$, where $L_{0}=20 \mathrm{~mm}$ and $c_{0}$ are specimen length and speed of sound, respectively, c.f. Table 1 .

The low noise level allows to use the average force expression, suitable to calculate the macroscopic stress in the specimen,

$F_{\text {avg }}=\frac{F_{L}+F_{R}}{2}$

Mohr et al. [24] have shown using simulations that this force estimate is the most accurate option to calculate the representative macroscopic stress from the strain gauge information. Here, the oscillations due to reverberation cancel out altogether, leading to an artefact-free and smooth average signal.

The evolution of dynamic equilibrium, i.e., the difference between left and right forces, is also well resolved. The equilibrium ratio,

$R=\frac{F_{L}-F_{R}}{F_{L}+F_{R}}$,

also displayed in Fig. 6, shows that dynamic equilibrium, i.e., $R<10 \%$ in this M150 foam specimen is reached after $\approx 6$ reverberations at a time of $\approx 0.4 \mathrm{~ms}$. The onset of plastic deformation can be identified form the yield peak at $t \approx 0.12$ $\mathrm{ms}$. This means that the foam specimen is not in equilibrium during compaction. For this reason, no constitutive behavior relevant for the bulk material can be inferred from this test, because we are essentially probing a non-equilibrium response which is governed by the large specimen size: The elastic wave speed is too low to establish equilibrium before yielding occurs.

Nevertheless, it is important to characterize this behavior. For practical applications such as impact protection, PU foams are used in thick layers. The Symmpact testing device enables a detailed perspective into such a loading scenario, as the next section will show.

\section{Results}

Fig. 7(A)-(C) shows stress/strain data obtained for foam specimens at three different nominal strain rates, which are equally spaced apart in logarithmic space: $\dot{\varepsilon}=[0.0017,0.5,500] / \mathrm{s}$. Five specimens were tested at each strain rate, but only representative results for two are shown in the graph to reduce clutter. The summarizing bar chart in Fig. 7(D) contains the mean and standard deviation estimate at selected values of strain calculated using all 5 specimens.

At the lowest engineering strain rate of 0.0017 /s, our measured values for strength and strain correspond well with the manufacturer's datasheet values listed in Table 1 . Increasing the loading rate from $0.0017 / \mathrm{s}$ to $0.5 / \mathrm{s}$ leads to a significant increase in strength by $\approx 30 \%$ for all foam types. Further increase to $500 / \mathrm{s}$ is accompanied by more strength for the higher-density foams M150 and M330. However, the same cannot be observed for the lowest-density foam M80.

Here, increasing the engineering strain rate from $0.5 / \mathrm{s}$ to 500 /s yields a rather peculiar effect: the entire stress/ strain curve at $500 / \mathrm{s}$ runs below the curve obtained at 0.5
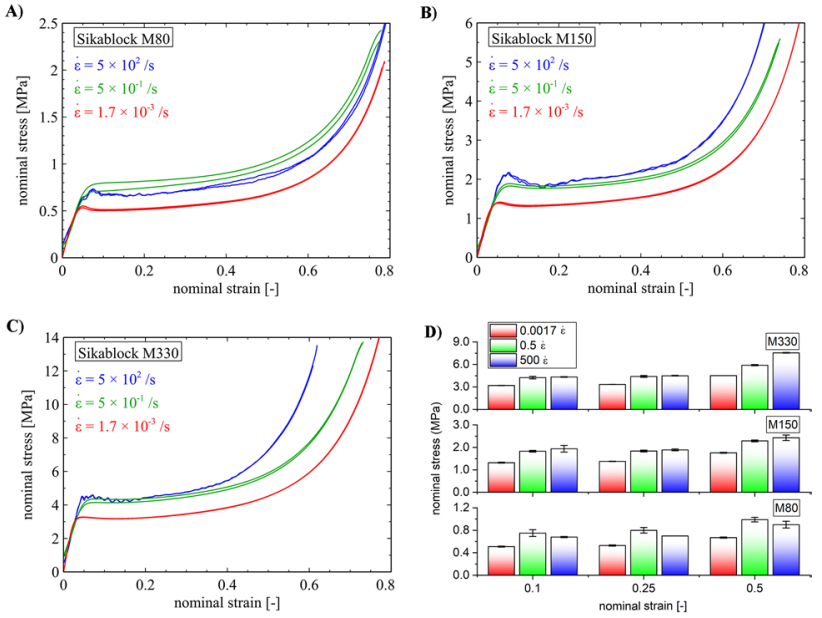

Fig. 7 (A) to (C) show the stress/strain data for the foams M80, $\mathrm{M} 150$, and M330 obtained at strains rates $\dot{\varepsilon}=0.0017 / \mathrm{s}, 0.5 / \mathrm{s}$, and $500 / \mathrm{s}$. (D) compares stress values at three selected strain values, $\varepsilon$ $=0.1,0.25$, and 0.5 , to emphasize the non-monotonic relation of stress to strain rate for the lower density foams M80 and M150 
/s. This effect is statistically significant compared to the scatter between individual experiments: the mean reduction in strength between these two strain rates is $0.1 \pm 0.04 \mathrm{MPa}$.

Thus, for the foams with higher densities, M150 and M330, a conventional positive strain rate sensitivity is observed. In contrast, foam M80, exhibit negative apparent strain rate sensitivity between the engineering strain rates $0.5 / \mathrm{s}$ and $500 / \mathrm{s}$. This is peculiar, as it is known that the base polymeric foam material, a polyurethane, typically exhibits a monotonic positive strain-rate sensitivity until much higher rates of strain, see e.g., [25]. However, our results are partially corroborated by [26], where a critical strain rate of $\approx$ 1000 /s was identified beyond which a different PUR foam lost strain rate dependency.

We will show in the following that the apparent negative strain rate sensitivity is a structural effect occurring at low foam density, caused by localization and inhomogeneity of the strain rate distribution in the specimen. To this end, the force equilibria according to equation (7) during high-rate loading, are plotted in Fig. 8. Dynamic equilibrium is achieved within $\approx 0.5 \mathrm{~ms}$ for the highest-density foam, M330, whereas M80 requires $\approx 1 \mathrm{~ms}$. Elastic wave reverberations can be identified for M330, but not for M80. As dynamic force equilibrium is linked to a homogeneous strain rate distribution via Newton's acceleration law, these observations indicate non-homogeneous deformations in the case of the lower density foams. This is further substantiated by resolving the local strain rate distribution in the specimen.

Figure 9 shows Lagrange diagrams of the three different foams compressed at nominally 500 /s. Here, fullfield strain rate measurements were made with DIC for every video frame recorded at $360 \mathrm{kHz}$. These fields are then averaged along the radial direction of the specimen,

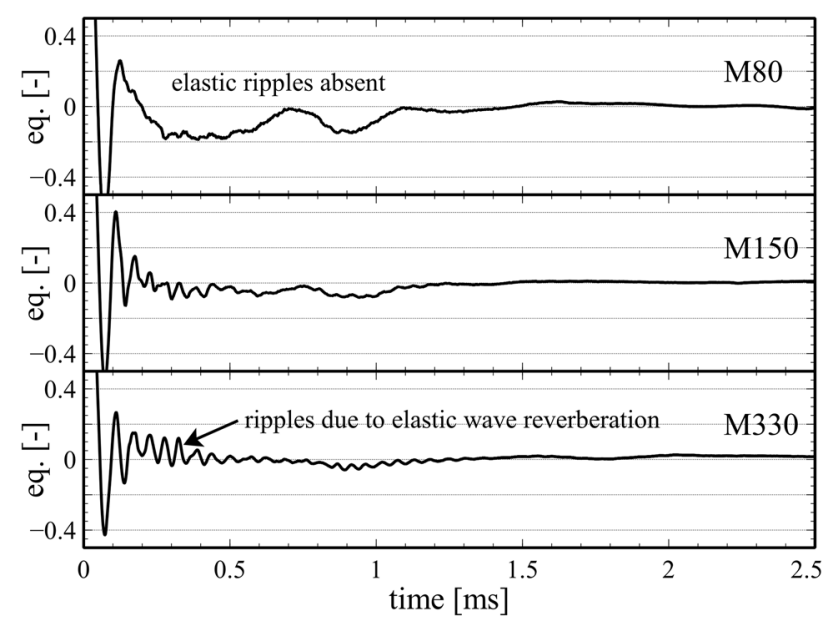

Fig. 8 Evolution of dynamic equilibrium during loading at an engineering strain rate of $500 / \mathrm{s}$

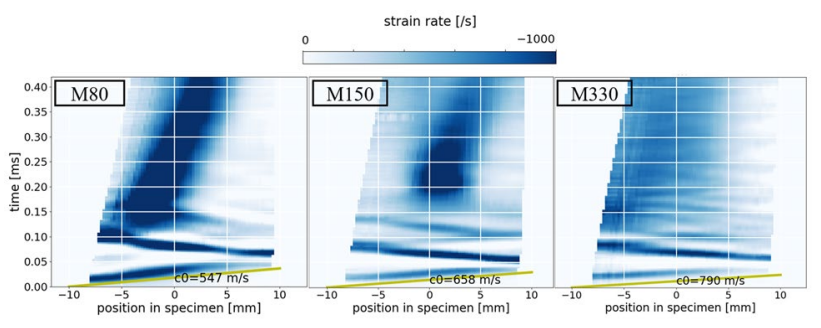

Fig. 9 Lagrange plots of strain rate distribution in the foam specimens during dynamic compression at an average strain rate of $500 / \mathrm{s}$. Yellow lines at the bottom indicate the elastic speed of sound taken from Table 1

assuming an axisymmetric deformation field. The resulting line plots of strain rate vs. longitudinal position are stacked vertically, yielding a Lagrange diagram where time increases on the vertical axis, and position on the horizontal axis. The local (both in space and time) amplitude of strain rate is visualized using color. For M80, we initially observe a compression front travelling through the specimen at elastic wave speed. The front is reflected on the right end and travels towards the left. Following this single reverberation, the strain rate amplitude localizes at $t=0.1 \mathrm{~ms}$ and forms a localized compression front, which travels to the right at a much reduced velocity of $\approx 10 \mathrm{~m} / \mathrm{s}$. For M150, we observe a similar pattern, but two reverberations instead of just one are visible. Strain rate localization then follows but is less pronounced. In contrast, for M330, a much more homogeneous strain rate distribution is observed with many visible wave reverberations.

The Lagrange diagram analysis agrees with the observations drawn from the evolution of force equilibration, c.f. Fig. 7: The low density foam M80 does not attain force equilibrium until it is significantly compacted. The reason for this becomes evident in the image analysis: instead of homogeneous strain rate distribution, we see localized collapse. For M330, on the other hand, force equilibrium is attained more quickly, and the strain rate distribution is much more uniform. M330 also shows a conventional positive strain rate dependency of strength. In contrast, the apparent strain rate sensitivity of M80 becomes negative at $500 / \mathrm{s}$, accompanied by structural collapse.

\section{Discussion and Conclusion}

This work provides detailed implementation of a directimpact Hopkinson bar with strain gauges on the input bar, which also serves as the striker. It is simple to build using off-the-shelf components. With its long pulse duration of $2.6 \mathrm{~ms}$ and polycarbonate as the bar material, it is suitable for dynamic compression experiments on low-impedance 
materials such as plastic foams. This work shows that force transients from strain gauges on the PC bars may be accurately obtained without viscoelastic corrections. However, the strain gauges need to be placed close to the specimen/ bar interface. Secondly, the non-linear elastic region of PC requires a quadratic calibration function, which maps Wheatstone bridge voltage readings to forces. Here, we use a specimen/bar interface to strain gauge distance of $130 \mathrm{~mm}$ and bars with a diameter of $40 \mathrm{~mm}$, resulting in estimated force measurement errors of less than $1 \%$ when compared to a reference dynamic load cell. For this particular setup, PC bars can be used just like metallic bars, but only if strain gauges are placed close to the specimen/bar interface. Similar observations were made by Rao et al. [27], albeit without quantifying the viscoelastic effects in detail as we did.

The setup presented here is particularly useful for investigating the evolution of dynamic force equilibrium. In contrast to conventional split Hopkinson pressure bars, the left- and right-travelling waves created upon the impact of the input bar on the specimen can be recorded directly without the problem of wave superposition, leading to comparatively accurate results for both the average force and dynamic force equilibrium.

We apply this measuring principle to a range of polyurethane foam specimens, which vary in density from 80 $-240 \mathrm{~kg} / \mathrm{m}^{3}$. These foams are peculiar in that a strain rate increase from $0.0017 / \mathrm{s}$ to $0.5 / \mathrm{s}$ yields an increase of strength by $\approx 30 \%$. However, a further increase of the strain rate to $500 / \mathrm{s}$ is not accompanied by a further increase of strength: the lowest-density foam even shows a decrease of strength. While we do not have data obtained at higher rates of strain, we note that Chen et al. [26] observed a related effect for rigid polyurethane foams. They found that strength and strain rate are linked at low rates of strain. In contrast, within the interval from 1000 /s to 5000 /s, no dependence of plateau strength on strain rate was observed, but the peak stress at yield increased with strain rate. However, their data cannot be directly compared to our data. Chen et al. [26] investigated very thin specimens with a length of $1.7 \mathrm{~mm}$ to achieve force equilibrium, while we tested specimens with $20 \mathrm{~mm}$ thickness to deliberately investigate a state of non-equilibrium.

In this work, we link the occurrence of a limit of the apparent strain rate sensitivity a transition from homogeneous deformation to structural collapse. Our sensitive measurement of lack of dynamic force equilibrium in low density foams supports the assumption of a discontinuity with different stress states downstream and upstream of the compaction front. In this regime, the foam is limited in its capability to further sustain stress because its structure breaks down locally. Our analysis shows that, for a real component designed to absorb impact loads, constitutive equations with monotonic strain rate models can be misleading. For the low density foam considered here, the apparent strain rate sensitivity for the $20 \mathrm{~mm}$ thick specimen is even negative. It is interesting to compare this effect observed in a foam to a solid polymer specimen, where the reverse behavior can be found if the specimen is chosen too large for dynamic equilibrium to occur: Dioh et al. [28] have shown in their 1995 work on SHPB tests of polyethylene samples, that specimens of thickness $4.4 \mathrm{~mm}$ exhibit an apparently higher yield stress than $1.5 \mathrm{~mm}$ thick specimens. The origin of this observation was tracked down to the existence of large stress and strain gradients which are more likely to occur in thick specimens than in small specimens - depending on the impact velocity. Dioh et al. conclude their work with the statement that at high strain measured flow or yield stress values must be shown to be independent of thickness if they are to be relied upon The work presented here allows drawing a related conclusion: The behavior of thick foam structures cannot be directly inferred from test data obtained from thin samples.

Acknowledgements P. Jakkula and G.C. Ganzenmüller gratefully acknowledge funding from Carl-Zeiss Foundation, grant title Skalenübergreifende Charakterisierung robuster funktionaler Materialsysteme. The authors declare no conflict of interest, in particular no financial or proprietary interests in any material discussed in this article.

Funding Open Access funding enabled and organized by Projekt DEAL.

Open Access This article is licensed under a Creative Commons Attribution 4.0 International License, which permits use, sharing, adaptation, distribution and reproduction in any medium or format, as long as you give appropriate credit to the original author(s) and the source, provide a link to the Creative Commons licence, and indicate if changes were made. The images or other third party material in this article are included in the article's Creative Commons licence, unless indicated otherwise in a credit line to the material. If material is not included in the article's Creative Commons licence and your intended use is not permitted by statutory regulation or exceeds the permitted use, you will need to obtain permission directly from the copyright holder. To view a copy of this licence, visit http://creativecommons.org/licenses/by/4.0/.

\section{References}

1. Song B, Chen W (2005) Split Hopkinson Pressure Bar Techniques for Characterizing Soft Materials. Lat Am J Solids Struct 2:113-152

2. Meyers MA (1994) Dynamic Behavior of Materials. John Wiley $\&$ Sons

3. Hopkinson B (1914) X. A method of measuring the pressure produced in thedetonation of high, explosives or by the impact of bullets. In: Philosophical Transactions of the Royal Society of London. Series A, Containing Papers of a Mathematical or Physical Character 213(497-508):437. https://doi.org/10.1098/ rsta.1914.0010 
4. Kolsky H (1949) An investigation of the mechanical properties of materials at very high rates of loading. Proc Phys Soc B 62(11):676-700. https://doi.org/10.1088/0370-1301/62/11/302

5. Zhao H, Gary G, Klepaczko JR (1997) On the use of a viscoelastic split HopkinsonPressure. Bar. Int J Impact Eng 19(4):319-230. https://doi.org/10.1016/S0734-743X(96)00038-3

6. Harrigan JJ, Ahonsi B, Palamidi E, Reid SR (2014) Experimental and numerical investigations on the use of Polymer Hopkinson Pressure Bars. Philos Trans Royal Soc A Math Phys Eng Sci 372(2023):20130201. https://doi.org/10.1098/rsta.2013.0201

7. Walley SM (2020) Highways and byways in the History of High Rate Mechanical Testing. J Dyn Behav Mater 6(2):113-158. https://doi.org/10.1007/s40870-020-00237-9

8. Chen W, Lu F, Zhou B (2000) A quartz-crystal-embedded split Hopkinson pressure bar for soft materials. Exp Mech 40(1):1-6. https://doi.org/10.1007/BF02327540

9. Govender RA, Curry RJ (2016) The "Open" Hopkinson pressure bar: Towards addressing force equilibrium in specimens with nonuniform deformation. J Dyn Behav Mater 2(1):43-49. https://doi. org/10.1007/s40870-015-0042-2

10. Hiermaier S, Meenken T (2010) Characterization of lowimpedance materials at elevated strain rates. J Strain Anal Eng Des 45(6):401-409. https://doi.org/10.1243/03093247JSA584

11. Lea LJ, Jardine AP (2016) Application of photon doppler velocimetry to direct impact Hopkinson pressure bars. Rev Sci Instrum 87(2):123101. https://doi.org/10.1063/1.4940935

12. Koumlis S, Lamberson L (2019) Strain rate dependent compressive response of open cell polyurethane foam. Exp Mech 59(7):1087-1103. https://doi.org/10.1007/s11340-019-00521-3

13. Barnes AT, Ravi-Chandar K, Kyriakides S, Gaitanaros S (2014) Dynamic crushing of aluminum foams: Part I - Experiments. Int J Solids Struct 51(9):1631-1645. https://doi.org/10.1016/j.ijsolstr. 2013.11.019

14. Ravindran S, Koohbor B, Malchow P, Kidane A (2018) Experimentalcharacterization of compaction wave propagation in cellular polymers. Int J Solids Struct 139-140:270-282. https://doi. org/10.1016/j.ijsolstr.2018.02.003

15. Tagarielli VL, Deshpande VS, Fleck NA (2008) The high strain rate response ofPVC foams and end-grain balsa wood. Compos Pt B Eng 39(1):83-91. https://doi.org/10.1016/j.compositesb.2007. 02.005

16. Lopatnikov SL, Gama BA, Jahirul Haque M, Krauthauser C, Gillespie JW, Guden M, Hall IW (2003) Dynamics of metal foam deformation during Taylor Cylinder-Hopkinson bar impact experiment. Compos Struct 61(1):61-71. https://doi.org/10.1016/S02638223(03)00039-4

17. Song B, Sanborn B, Lu WY (2019) Experimental measurement and analysis of stress/shock wave propagation speed through pre-strained silicone foam pads under lateral confinement. J Dyn Behav Mater 5(2):170-179. https://doi.org/10.1007/ s40870-019-00196-w

18. Hoffmann K (2001) Applying the Wheatstone Bridge Circuit. Hottinger Baldwin Messtechnik, Darmstadt, Germany (W1569-1.0 en)

19. Safford N (1988) High strain rate studies with the direct impact Hopkinson bar. University of Cambridge (PhD thesis)

20. Rey Calderón AA, Díaz Díaz A (2018) New aspects in the mechanicalbehavior of a polycarbonate found by an experimental study. Adv Mater Sci Eng 2018:1540919. https://doi.org/10.1155/ 2018/1540919

21. Davis JL (1964) An elementary theory of nonlinear viscoelasticity. J Polym Sci Pt A Gen Pap 2(3):1311-1320. https://doi.org/10. 1002/pol.1964.100020328

22. Bacon C (1998) An experimental method for considering dispersion and attenuation in a viscoelastic Hopkinson bar. Exp Mech 38:242-249

23. Curry RJ, Cloete T, Govender R (2012). Implementation of viscoelastic Hopkinson bars. EPJ Web Conf. https://doi.org/10.1051/ epjconf/20122601044

24. Mohr D, Gary G, Lundberg B (2009) Theoretical assessment of stress-strain curve estimates in split Hopkinson bar experiments. In DYMAT - International Conference on the Mechanical and Physical Behaviour of Materials under Dynamic Loading, vol.1 (EDP Sciences), vol. 1, pp. 485-490. https://doi.org/10.1051/ dymat/2009069

25. Sarva SS, Deschanel S, Boyce MC, Chen W (2007) Stress-strain behavior of a polyurea and a polyurethane from low to high strain rates. Polymer 48(8):2208-2213. https://doi.org/10.1016/j.polymer. 2007.02.058

26. Chen W, Lu F, Winfree N (2002) High-strain-rate compressive behavior of a rigid polyurethane foam with various densities. Exp Mech 42(1):65-73. https://doi.org/10.1007/BF02411053

27. Rao S, Shim VPW, Quah SE (1997) Dynamic mechanical properties of polyurethane elastomers using a nonmetallic Hopkinson bar. J Appl Polym Sci 66(4):619-631. https://doi.org/10.1002/ (SICI)1097-4628(19971024)66:4619::AID-APP23.0.CO;2-V

28. Dioh NN, Ivankovic A, Leevers PS, Williams JB (1995) Stress wave propagationeffects in split Hopkinson pressure bar tests. Proc Math Phys Sci 449(1936):187-204

Publisher's Note Springer Nature remains neutral with regard to jurisdictional claims in published maps and institutional affiliations. 